\title{
Intelligent Techniques for Fed-Batch Bioprocess Control
}

\author{
MIHAI CARAMIHAI, IRINA SEVERIN \\ University POLITEHNICA Bucharest, \\ Spl. Independentei, 313, sector VI, Bucharest, Romania \\ ROMANIA \\ m.caramihai@ieee.org, irina_severin2003@yahoo.com
}

\begin{abstract}
Bioprocesses are appreciated as difficult to control because their dynamic behavior is highly nonlinear and time varying, in particular, when they are operating in fed batch mode. For this kind of bioprocess where the mathematical model contains many structured and unstructured uncertainties, we try to combine different intelligent techniques based on natural syllogisms of these techniques. In order to obtain a high bioprocess productivity it is essential to accord the benefits of the classical control strategy (i.e. the analytical determination of the optimum) with the subjective bioprocess characterization (due to the human expert) in order to diminish the on line information scarcity. The research objective of this study was to develop an appropriate control method for a new complex bioprocess and to implement it on a laboratory plant. Hence, an intelligent control structure has been designed in order to produce biomass and to maximize the specific growth rate..
\end{abstract}

Key-Words: - bioprocess, fed batch; mass-balance model; intelligent techniques, fuzzy control

\section{Introduction}

Bioprocesses are appreciated as difficult to control because their dynamic behavior is highly nonlinear and time varying, in particular, when they are operating in fed-batch mode. That is why they are interesting testing benches for non-linear and intelligent control techniques. Several techniques have been proposed [1-3] and tested by simulation, but only few have been implemented on real bioprocesses.

There are two main reasons: at first, it is difficult to get a "good" model of the process which is experimentally validated in order to design the control algorithm; and then, the available on line instrumentation on a bioprocess is generally poor, there are only few sensors to deliver on line measurements of state variables required for control implementation.

\section{Problem Formulation}

The state of the art in the bioprocess control systems comprises three main control strategies:

(2) The classic control strategy based on a priori model describing the global evolution of the bioprocess, but due to the scarce bioprocess information, the model not being able to represent the whole bioprocess behavior

(2) The control strategy based on adaptive techniques, without a global optimization ability (i.e. the bioprocess is optimized during a simple period)

- The control strategy based on intelligent techniques, which uses human subjective expert knowledge, but a general optimal procedure cannot be standardized.

In order to obtain a high bioprocess productivity it is essential to accord the benefits of the classical control strategy (i.e. the analytical determination of the optimum) with the subjective bioprocess characterization (due to the human expert) for the rejection of the on line information scarcity.

The research objective of this study was to develop an appropriate control method for a complex bioprocess and to implement it on a laboratory plant, namely the control of the fed batch cultivation of Hansenula polymorpha yeast for alcoholoxidase-containing biomass. At first, the process is described and a new mathematical model is proposed and then the control strategy is defined and the intelligent control structure is designed. Finally, the control performances are tested through real data.

\section{Paper approach}

\subsection{Methodology}

A discontinuous fed-batch bioprocess for alcoholoxidase-containing biomass with the methylotrophic yeast Hansenula polymorpha CBS 4732 was operated in an airlift lab - bioreactor. The intracellular enzyme, to be separated further on, is used for obtaining a high-specialized kit for methanol/ethanol determination. The yeast was cultivated on a complex medium with $\left(\mathrm{NH}_{4}\right)_{2} \mathrm{SO}_{4}, \quad \mathrm{KH}_{2} \mathrm{PO}_{4}, \quad \mathrm{Na}_{2} \mathrm{HPO}_{4}$, $\mathrm{MgSO}_{4} * 7 \mathrm{H}_{2} \mathrm{O}, \mathrm{CaCl}_{2}$, yeast extract or autolysed residual 
beer yeast as organic $\mathrm{N}$ source and microelements (Fe, $\mathrm{B}, \mathrm{Cu}, \mathrm{I}, \mathrm{Mn}, \mathrm{Zn}, \mathrm{Mo}$ ).

$$
\begin{aligned}
& \frac{d V}{d t}=-\frac{E_{S}}{\rho_{S}}-\frac{E_{M}}{\rho_{M}} \\
& \frac{d X}{d t}=\frac{\mu_{\max } S}{K_{S}+S} X+\frac{X}{V}\left(\frac{E_{S}}{\rho_{S}}+\frac{E_{M}}{\rho_{M}}\right) \\
& \frac{d S}{d t}=-\frac{\mu_{\max } S}{K_{S}+S} \frac{X}{Y_{X / S}}-\frac{E_{S} \rho_{S}}{V}+\frac{S}{V}\left(\frac{E_{S}}{\rho_{S}}+\frac{E_{M}}{\rho_{M}}\right)
\end{aligned}
$$

where: $E_{S}$ and $E_{M}$ are the substrate and medium loss by evaporation $[\mathrm{g} / \mathrm{h}] ; \rho_{\mathrm{S}}$ and $\rho_{\mathrm{M}}$ are the substrate and medium densities $[\mathrm{g} / \mathrm{L}] ; \mathrm{Y}_{\mathrm{X} / \mathrm{S}}$ is the substrate conversion yield referred to the biomass [g dry matter/g substrate]; $\mu$ is the specific growth rate $[1 / \mathrm{h}] ; \mathrm{V}$ is the volume of the cultivation medium in the bioreactor [L]; $\mathrm{X}$ and $\mathrm{S}$ are the biomass and substrate concentrations $[\mathrm{g} / \mathrm{L}]$ and $t$ is the time [h], $\mu_{\max }$ represents the maximum specific growth rate $[1 / \mathrm{h}]$ and $\mathrm{K}_{\mathrm{S}}$ is the saturation constant $[\mathrm{g} / \mathrm{g}]$. The main process parameters were: continuous temperature control $37^{\circ} \mathrm{C}$; a minimal level of $\mathrm{pO}_{2}-10 \%$ from the saturation concentration was maintained during the exponential growth; continuous $\mathrm{pH}$ control between 4.5 5.0 by addition of $\mathrm{NH}_{4} \mathrm{OH}(12.5 \%)$; no foam control, if the main parameters are optimally controlled. The unique $\mathrm{C}$ source, the methanol was introduced function of the yeast growth rate in connection with the substrate consumption rate for avoiding the growth inhibition by substrate concentration. The developed model (1) is based on the mass-balance principle and on the hypothesis of a non-inhibitive substrate effect (i.e. the specific growth rate is defined by the Monod equation). In line with the operation mode (fed-batch with discontinuous substrate feeding), there are discontinuous variations of the main variables due to: substrate feeding, medium feeding (to overcome the loss by evaporation or sample collection) or samples withdraws. That is why the following mass-balance equations are to be added to express each discontinuous modification for volume, and substrate or biomass concentrations

$$
\begin{aligned}
& V_{k}+A_{S k}+A_{M k}=P_{M k}+V_{k+1} \\
& S_{k} \rho_{M} V_{k}+A_{S k} \rho_{S}=P_{M k} \rho_{M} S_{k}+S_{k+1} \rho_{M} V_{k+1} \\
& X_{k} V_{k}=P_{M k} X_{k}+X_{k+1} V_{k+1}
\end{aligned}
$$

where: $\mathrm{Vk}, \mathrm{Vk}+1=$ volume before $/$ after modification $[\mathrm{L}] ; \mathrm{ASk}, \mathrm{AMk}=$ substrate volume and respectively medium volume adding [L]; PMk=sample withdraw [L]. The same notations are used for Sk, Sk+1 and $\mathrm{Xk}, \mathrm{Xk}+1$. We use: $\rho \mathrm{S}=800[\mathrm{~g} / \mathrm{L}]$., respectively $\rho \mathrm{M}=1000[\mathrm{~g} / \mathrm{L}]$. The identification of the model parameters was carried out based on measured values in order to minimize the modeling error. The identification procedure (i.e. Nelder-Mead algorithm) determines the optimum values for the following process parameters: $E S, E M, \mu \max , K_{S}$ and $\mathrm{Y}_{\mathrm{X} / \mathrm{S}}$.

\subsection{Experimental arrangement}

The separate identification of the model parameters (decoupling of the model equations) was possible because the equation that describes the variation of the cultivation medium depends only on two parameters, the medium and substrate loss by evaporation. Based on the experimental data, in the first step, the identification procedure was able to determine the optimal values of the medium and substrate loss by evaporation. In the second identification step, the procedure determines the optimal values of the other remaining model parameters. The simulation results of the last two equations from (1) are presented in Figure 1, in comparison with the two experimental data sets.
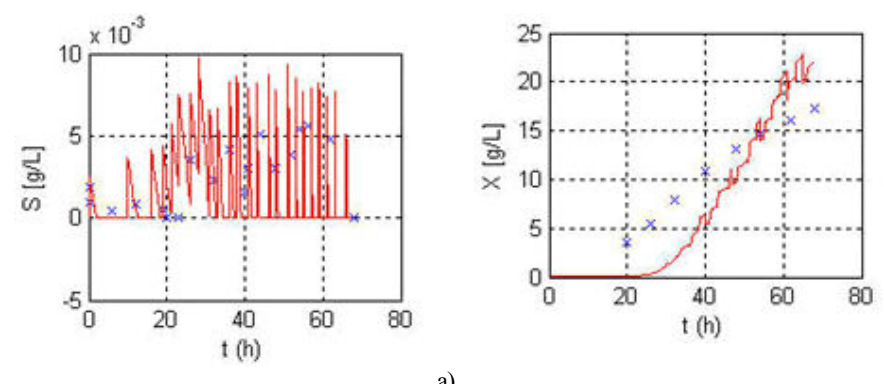

a)
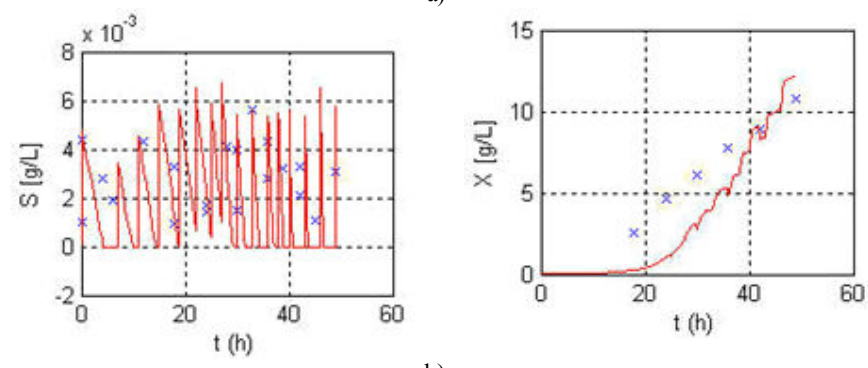

b)

Fig. 1. Simulation results of the substrate and medium concentrations a) first experiment; b) second experiment; ('--' - simulation, ' $x$ ' - experimental data)

The agreement between real and modeling data is good enough to allow the obtainment of the optimal substrate concentration for the growth process control. The identification results show the mathematical model closely follows the experimental data.

\section{A case study}

For this bioprocess, the overall control objective is to obtain large biomass quantities, based on the assumption 
that high biomass concentration will assure the obtaining of important alcoholoxidase-active biomass. In this paper a control system based on fuzzy logic is proposed. It is well known that Fuzzy Control Systems (FCS) can manipulate incomplete and uncertain information about the process assuring high control performances [4-6]. The proposed FCS receives information about the state of the bioprocess expressed by the biomass and substrate concentrations. Based on this information, FCS computes the quantity of substrate to be added into the reactor. According to these observations the inputs of FCS are the biomass (X) and substrate (S) concentrations, and the output is the correction to be applied on the substrate addition. The rules of FCS are presented in Table 1.

\section{Table 1. The rule base}

\begin{tabular}{|l|r|r|r|}
\hline $\boldsymbol{S}_{\boldsymbol{k}} \boldsymbol{X}_{\boldsymbol{k}}$ & $\boldsymbol{S}$ & $\boldsymbol{M}$ & $\boldsymbol{L}$ \\
\hline $\boldsymbol{S}$ & $\mathbf{Z}$ & $\mathbf{P Z}$ & $\mathbf{P}$ \\
\hline $\boldsymbol{M}$ & $\mathbf{N Z}$ & $\mathbf{Z}$ & $\mathbf{P Z}$ \\
\hline $\boldsymbol{L}$ & $\mathbf{N}$ & $\mathbf{N Z}$ & $\mathbf{Z}$ \\
\hline
\end{tabular}

Rules evaluation by the inference engine is made according to the min-max inference rule and the output defuzzyfication is made based on the centroid defuzzyficaton method.

The control loop was implemented in MATLAB, version 8.5. For control loop simulation the proposed mathematical model was used and the simulation results were compared with the experimental data.
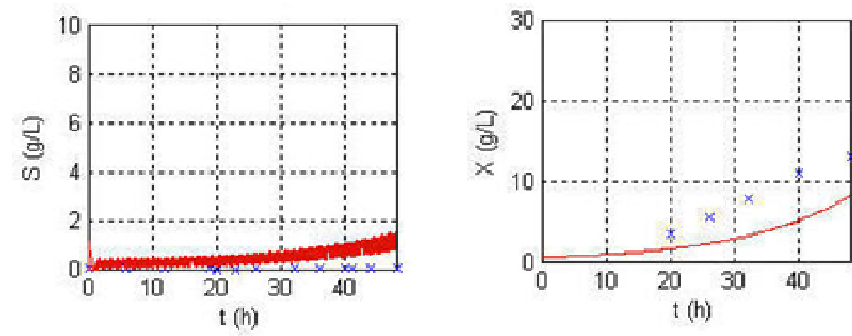

a)
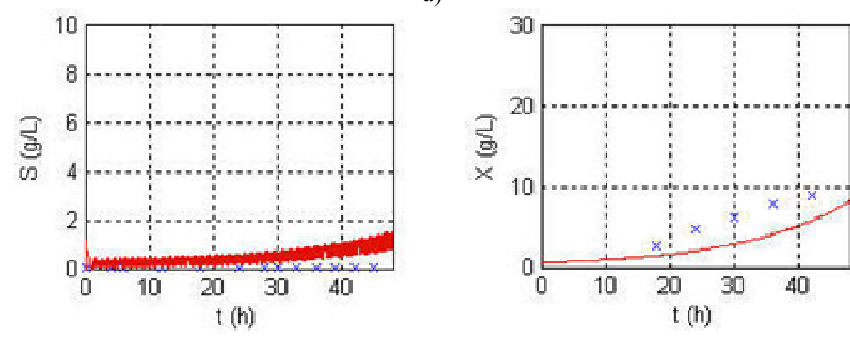

b)

Fig. 2. Simulation results of the control loop: a) first experiment; b) second experiment; ('-' - simulation results; ' $x$ ' - experimental data)
The simulation results show that the proposed fuzzy control system is capable of computing the substrate feedings needed for cell growth according to the biomass concentration increase. The evolution of the substrate concentration marks the substrate consumption and additions, as well as the increase of the additions along with cell growth. The biomass concentration obtained by simulation follow closely the experimental data.

\section{Conclusions}

An intelligent control structure has been designed for a complex bioprocess in order to maximize the biomassproducing enzyme formation rate. Three sets of experimental data were used to test and validate with good results the mathematical model and further on the control system. It is to be observed that the success of control implementation is critically dependent upon the technical operating conditions of the process.

\section{References:}

[[1] R. Oliveira, Comp. \& Chem. Eng., 28 (2009), 755

[2] M. Jench, S. Gnoth, M. Beck, M. Kleinschmidt, R. Simutis, A. Lubbert, J. Biotech, 127 (2006), 84

[3] C. Komives, R. S. Parker, Curr. Opinion Biotech, 14(2008), 468

[4] F. Renard, A. et all, J. Process Contr., 2009

[5] E. K. Junso, J. Knolof, 16th IFAC W. Congress, Prague, 2005, Topic 84

[6] K. Schugerl, K. H. Bellgardt, Bioreact. Eng, Springer Verlag, Berlin, 2000 